\title{
Roland Parenteau
}

Président du Conseil d’orientation économique du Québec

\author{
(1964)
}

\section{"Les régions riches et les régions pauvres du Québec”}

Un document produit en version numérique par Michel Fortin, bénévole,

Adjoint à la mairie, Ville de Saguenay, province de Québec

Courriel: micfortin@videotron.ca ou micfor@ville.chicoutimi.qc.ca

Dans le cadre de: "Les classiques des sciences sociales"

Une bibliothèque numérique fondée et dirigée par Jean-Marie Tremblay, professeur de sociologie au Cégep de Chicoutimi

Site web: http://classiques.uqac.ca/

Une collection développée en collaboration avec la Bibliothèque

Paul-Émile-Boulet de l'Université du Québec à Chicoutimi

Site web: http://bibliotheque.uqac.ca/ 
Cette édition électronique a été réalisée par Michel Fortin, bénévole, adjoint politique, Ville de Saguenay, à partir de :

\section{Roland Parenteau}

[Président du Conseil d’orientation économique du Québec].

“Les régions riches et les régions pauvres du Québec”.

Un article publié dans la revue Cité libre, $45^{\mathrm{e}} 16$ octobre 1964, pp. 6-12.

Polices de caractères utilisée :

Pour le texte: Times New Roman, 14 points.

Pour les citations : Times New Roman, 12 points.

Pour les notes de bas de page : Times New Roman, 12 points.

Édition électronique réalisée avec le traitement de textes Microsoft Word 2004 pour Macintosh.

Mise en page sur papier format : LETTRE (US letter), 8.5'” x 11’')

Édition numérique réalisée le 26 février 2007 à Chicoutimi, Ville de Saguenay, province de Québec, Canada. 


\section{Table des matières}

$\underline{\text { Introduction }}$

1. Aperçu historique

1.1. Mise en valeur du territoire avant la période d'industrialisation

1.2. $\underline{\text { La mise en valeur du territoire à l'époque de l'industrialisation }}$

2. La situation présente des régions

2.1. La région de Montréal

2.2. Les régions diversifiées à centre urbain

2.3. Les régions périphériques

3. Les attitudes possibles 
Roland Parenteau 1

[Président du Conseil d’orientation économique du Québec].

“Les régions riches et les régions pauvres du Québec”.

Un article publié dans la revue Cité libre, 45e 16 octobre 1964, pp. 6-12.

\section{Introduction}

La prise de conscience des déséquilibres entre régions d'un même espace politiquement homogène représente une nouvelle préoccupation qui fait étrangement contraste avec la mentalité traditionnelle qui tenait à tout prix à considérer un pays comme un espace pratiquement indifférencié, au point qu'une impulsion quelconque donnée en un point ou l'autre du territoire qu'elle fut d'origine publique ou privée devait exercer ses effets indifféremment en tous les points de ce territoire.

On raisonnait en d'autres termes à l'intérieur d'un schéma de concurrence pure, dans lequel les facteurs de production se trouvaient parfaitement mobiles. Conséquence logique : il était impensable que des disparités notables s'introduisent dans le système, puisque les mécanismes du marché étaient là pour veiller au grain et niveler les conditions de développement. En définitive donc l'implantation des activités économiques, le choix des localisations devaient être conditionnées par la richesse relative des diverses régions. Notons cependant qu'un tel raisonnement s'appliquait uniquement aux aires géographiques nationales, comme si les échan-

1 Roland Parenteau, « Les régions riches et les régions pauvres du Québec », dans Cité Libre, 16 octobre 1964, pp. 6-12. 
ges de biens et de capitaux changeaient de nature quand ils s'effectuent entre pays différents.

Tout le monde aura compris que les théories auxquelles je réfère actuellement sont les théories économiques qui ont été à l'honneur jusqu'à ces toutes dernières années. Je ne voudrais pas toutefois insinuer que les conceptions punctiformes des économistes étaient partagées par leurs chers collègues géographes et sociologues. Ceux-ci en effet sont depuis toujours conscients de l'existence des régions avec des personnalités très disparates, des taux de croissance différents, des conditions de développement très diverses.

Il faudrait ajouter à la décharge des économistes que pendant longtemps les statistiques faisaient terriblement défaut qui auraient permis de mesurer les disparités régionales et de prendre ainsi conscience de l'imperfection des schémas d'analyse traditionnels trop centrés sur l'espace national. Mais on pourrait répondre que la coexistence, pas toujours pacifique, des peuples riches et des peuples pauvres pendant des siècles aurait dû susciter des réflexions salutaires sur le bien-fondé des théories dominantes. Expliquer des disparités aussi radicales de développement que celles que l'on constatait entre pays par des différences trop accentuées dans les mentalités ou les capacités intellectuelles, ou encore l'inégale répartition des richesses naturelles pouvait suffire à l'esprit pressé d'une certaine époque mais ne pouvait résister longtemps à un effort d'analyse le moindrement sérieux.

Quoi qu'il en soit, la mode actuelle est aux études régionales et tant mieux pour la réputation des économistes. C'est d'ailleurs précisément la préoccupation toute nouvelle de favoriser la croissance des économies retardées qui explique cette orientation récente de la science économique vers les études régionales. Et l'on est vite passé des disparités entre pays ou même continents aux disparités entre régions d'un même pays. Car on s'est aperçu qu'à l'intérieur même des pays les plus évolués, des divergences sensibles se manifestaient, divergences que l'opinion est de moins en moins portée à accepter, surtout celle des régions défavorisées. Ainsi pour prendre l'exemple du Canada, le revenu personnel par tête à Terre-Neuve 
se trouve exactement la moitié de celui de l'Ontario, et de la Colombie-Britannique et cela, remarquez le bien, une fois que les multiples politiques de transfert ont exercé leurs effets supposés compensateurs.

Au Canada, le problème des disparités régionales peut être envisagé à deux niveaux : d'abord celui de la grande région, i.e. les cinq grandes régions du Canada, entre lesquelles des différences profondes apparaissent tant du point de vue de la vocation naturelle que des rythmes de développement et des degrés de prospérité.

Mon intention, ici, n'est pas de commenter cet aspect de la question, mais d'étudier plutôt le problème au niveau de la petite région, c'est-à-dire des diverses parties constituantes de l'une de ces grandes régions : le Québec. Considérer en effet, la province de Québec comme un ensemble homogène sous prétexte qu'une même culture prédomine partout et qu'une même autorité politique y exerce son influence également serait fort éloigné de la réalité. C'est aussi douteux que de parler d'un pseudo-marché du Québec, laissant supposer l'existence d'une certaine cohésion interne des échanges à l'intérieur de la province. Je me propose donc d'analyser ce phénomène, laissant à d'autres le soin d'étudier des aspects particuliers et de proposer les éléments d'une politique régionale. Ma compétence particulière m'amènera à mettre l'accent sur les aspects économiques, ce qui ne veut pas dire que je sous-estime les autres aspects, culturel, sociologique, etc., de la question.

\section{APERÇU HISTORIQUE}

$\underline{\text { Retour à la table des matières }}$

Quelques considérations tirées de l'histoire nous permettront de mieux comprendre la situation actuelle. Celle-ci en effet n'a pas toujours prévalu. Il y a lieu à cet égard de distinguer dans les méthodes de mise en valeur du territoire deux époques tout à fait différentes l'une de l'autre. Loin de moi l'intention de laisser entendre qu'on est 
passé brusquement d'un type de mise en valeur à l'autre. En fait, la transition a été graduelle nais les découpages historiques ont tout de même l'avantage de mieux faire ressortir les caractéristiques d'une époque. En l'occurrence, il s'agit de décrire deux types, deux « patterns » de développement économique. Dans les deux cas d'ailleurs, l'occupation du territoire s'est faite de façon tout à fait incoordonnée, sans planification préalable, en laissant agir les initiatives privées comme bon lui semblait.

\subsection{Mise en valeur du territoire avant la période d'industrialisation}

$\underline{\text { Retour à la table des matières }}$

Dans un premier stade, la mise en valeur du territoire s'est faite à partir de la vallée du Saint-Laurent et des autres vallées de la Province par processus de propagation qui faisait essaimer colons des centres déjà développés et parfois ombrés vers les régions nouvelles mais limitrophes. C'est en grande partie sous la pression démographique d'un taux de naissance exceptionnellement élevé que des individus entreprenants voyaient ainsi forcés d'aller occuper de nouveaux territoires. C'était là la seule alternative à l'absorption des populations excédentaires par les zones urbaines, absorption qui prenait la forme à cette époque d'une émigration vers la Nouvelle-Angleterre.

L'activité économique de cette époque était caractérisée surtout par une agriculture vivrière combinée d'ailleurs avec l'exploitation forestière sur une base de petites entreprises. C'était le type même de mise en valeur extensive, exigeant de vastes espaces, et cela d'autant plus que, toutes les terres n'étant pas riches, on était parfois obligé de sauter par-dessus des étendues de terrain considérables pour aller rejoindre les zones plus favorables à l'agriculture, et que les méthodes de culture se révélaient très rudimentaires et par conséquent, de faible productivité. Le résultat net de ce type de mise en valeur, auquel se mêlait d'ailleurs une sorte de frénésie de mettre la main — j'allais dire la hache - sur l'ensemble du territoire transparaît dans la contexture économique et sociale de la province : à savoir un peu- 
plement discontinu, de faible densité, avec des noyaux de population importants séparés les uns des autres par d'immenses déserts.

D'autres activités économiques se sont sans doute greffées sur l'agriculture, en particulier l'industrie et le commerce, mais elles étaient surtout de caractère rural et étaient poursuivies par la petite entreprise artisanale. C'était l'époque de l'économie de village, les artisans des petites agglomérations fournissant certains produits manufacturés aux cultivateurs des environs, ceux-ci par contre acheminant les denrées alimentaires vers le village. Économie vraiment autochtone dans laquelle peu d'échanges s'effectuaient avec l'extérieur et cela d'autant plus que les moyens de transport étaient relativement peu développés au point même qu'en hiver les principales voies de communication se trouvaient complètement fermées. Peu de complications au sujet de la localisation des activités industrielles et commerciales à cette époque de marchés géographiquement limités. L'industrie était d'initiative locale, les ressources de toutes sortes puisées sur place. On ne se posait pas la question de savoir où elle s'implanterait ; elle s'implantait en réalité dans le village du fondateur.

Il existait toutefois dans la province, mais un peu en marge du reste de l'économie, deux grands centres commerciaux, Québec et Montréal qui avaient l'avantage d'être des ports de mer importants et par lesquels s'effectuaient les transactions avec l'étranger. Les fonctions de Montréal à l'époque cependant étaient beaucoup plus commerciales qu'industrielles.

Quelques-uns se poseront peut-être la question suivante : pourquoi insister sur cette période à jamais révolue et dans laquelle la présente génération ne se reconnaît guère ? La réponse est assez simple. C'est que justement cette période n'est pas si révolue que cela, ou du moins elle l'est en ce sens que le développement économique obéit désormais à d'autres lois ; mais par ailleurs les conséquences économiques et sociales de ce type de mise en valeur se font toujours sentir et constituent notre héritage économique actuel ; en d'autres termes une grande partie des déséquilibres que nous connaissons présentement vient précisé-ment de la persistance à côté d'un secteur moderne de ces vestiges d'économie archaïque qui n'a pas été entièrement supplanté par des initiatives de fraîche date. 


\subsection{La mise en valeur du territoire à l'époque de l'industrialisation}

Je vous fais grâce de la description détaillée de l'évolution économique depuis la période à laquelle je viens de faire allusion jusqu'à une époque plus récente. Disons simplement qu'à partir de la fin du XIXe siècle, l'économie de la province de Québec s'est industrialisée en ce sens qu'on y a vu apparaître l'industrie manufacturière d'une certaine taille. Les établissements, dans certains cas, résultaient tout simplement de la croissance naturelle des entreprises existantes; dans d'autres cas, ils étaient dus à l'initiative de capitaux étrangers, j'entends étrangers à la région. Cette période, caractérisée par la réduction relative de l'agriculture au bénéfice de l'industrie de transformation et l'agrandissement incessant des marchés, compte énormément dans une première phase sur l'abondance et le bon marché de la main-d'œuvre et la proximité relative des matières premières. Ces facteurs de localisation caractéristiques de la fin du XIXe siècle s'accommodaient d'une certaine décentralisation géographique qui permettait à une multitude d'entreprises de moyenne envergure de s'installer dans les villes existantes. Du seul fait de leur installation d'ailleurs, une polarisation d'activités s'ensuivait, déclenchant un processus de croissance urbaine.

À une époque plus récente, cependant, la concentration technique de la fabrication avec tous les avantages qu'elle comporte sur le plan des coûts, combinée avec des techniques de vente permettant d'approvisionner des marchés de plus en plus étendus, a amené une réduction sensible du nombre des entreprises au point que, dans certains cas, une seule usine est en mesure d'approvisionner l'ensemble du marché. Il était alors fatal que cette concentration technique aille de pair avec une concentration géographique.

À partir du moment, en effet, où il faut réunir en un seul établissement la totalité ou la presque totalité de la production, on songe tout 
naturellement à installer l'usine en question tout près du marché le plus important. Ce sont des agglomérations déjà développées, arrivées à un certain point de maturité qui sont ainsi favorisées. Ces agglomérations réunissent à la fois un nombre considérable de consommateurs éventuels, mais aussi une main-d'œuvre diversifiée susceptible de satisfaire aux demandes les plus exigeantes. À l'époque actuelle, un effet complémentaire se dessine, accentuant encore la concentration. En effet, le perfectionnement des méthodes commerciales transforme les grandes agglomérations en centres de regroupement des produits, de telle sorte que ce sont les producteurs les plus rapprochés qui sont favorisés.

D'abord les entreprises modernes de grande envergure sont intéressées, dans la mesure où elles ont tendance à se spécialiser de plus en plus, à multiplier les liaisons techniques avec d'autres entreprises de même calibre, à disposer à proximité d'un pool de techniciens de toutes sortes et de moyens d'études et de recherches. De plus ces entreprises à marché national sont aussi préoccupées de minimiser le coût du transport de leurs produits et à rechercher par conséquent la proximité des agglomérations d'où l'on peut facilement rayonner que ce soit par chemins de fer, par route ou par avion.

Nous voilà donc en présence d'un phénomène cumulatif par lequel les entreprises s'installant dans les endroits où existent déjà à la fois une certaine concentration des activités économiques et une certaine diversification déclenchent un processus de croissance de nature à rendre encore plus accueillant à tous les points de vue, le milieu géographique concerné. 


\section{LA SITUATION PRÉSENTE DES RÉGIONS}

$\underline{\text { Retour à la table des matières }}$

Du point de vue économique, l'héritage que nous a légué l'histoire est le suivant. Juxtaposition de régions anciennes qui se trouvent à l'heure actuelle stagnantes ou en perte de vitesse avec d'autres régions qui sont sinon nouvelles, du moins beaucoup plus dynamiques puisqu'elles polarisent la plupart des activités nouvelles d'une certaine envergure. Je n'aurai pas l'outrecuidance de vous proposer une division de la Province en régions économiques. Mais on peut quand même distinguer, en s'en tenant aux traits principaux quatre types de régions.

\subsection{La région de Montréal}

Voilà en réalité la seule agglomération vraiment diversifiée, le seul centre polarisateur d'activités, c'est-à-dire capable d'engendrer par lui-même des activités nouvelles. C'est une région qui peut être qualifiée dans le plein sens du terme de région motrice. Elle possède en effet à un degré éminent à peu près tous les facteurs de localisation, qu'ils soient d'ordre psychologique ou d'ordre physique, les quelques éléments qui lui manquent comme les matières premières ou l'énergie électrique ou d'autres facteurs de production pouvant être facilement importés à bon compte. Je ne veux pas dire par là que Montréal constitue en toutes circonstances la localisation idéale. Loin de là. Bien qu'il faille constater que pour une multitude de chefs d'entreprise, ce soit l'opinion prédominante. Le fait que cette attitude manque quelque peu de rationalité ne change rien à la question. Il s agit ici de prendre connaissance des attitudes les plus générales et d'en mesurer les effets sur la croissance plus ou moins équilibrée ces régions. 
En plus des facteurs traditionnels de localisation, maind'œuvre, capitaux, matières premières, disponibilité de techniciens ou d'administrateurs d'entreprises, la région montréalaise offre un milieu social et une vie culturelle qui sont sans doute le résultat de l'importance de l'agglomération mais aussi une cause non négligeable de fixation de l'activité économique. L'on se rend compte en effet, de plus en plus, que les activités de type moderne exigeant une proportion considérable de techniciens ayant atteint un niveau culturel élevé, doivent être localisés à proximité d'un centre d'une certaine envergure qui seul peut offrir ces avantages. C'est ce qui explique non seulement la concentration dans le noyau urbain de beaucoup d'établissements, mais la constitution d'une constellation de satellites autour du grand centre, satellites qui ont la particularité d'offrir à la fois les avantages de la grande ville et ceux de la moyenne. Ajoutons à cela que Montréal étant la seule ville vraiment cosmopolite de la province de Québec, c'est celle où les étrangers se sentent plus à l'aise, facteur qui n'est pas négligeable dans la mesure où l'initiative en matière de développement industriel sera précisément due aux étrangers.

Tout cela est tellement vrai que dans l'ensemble de la province, Montréal est la seule ville où une exposition universelle puisse avoir lieu. Et même sans aller aussi loin, l'on peut dire que c'est la seule ville de la province où les congrès réunissant quelque milliers de personnes peuvent gendre place. L'administration des entreprises modernes exige de la part de leurs dirigeants des relations fréquentes avec les autres grands centres continentaux comme New-York et Toronto. Montréal se trouve, précisément sur ce point, bien desservie par les moyens de communication modernes comme l'avion, qui permettent à des membres de l'état-major des entreprises, d'effectuer des voyages d'affaires aller-retour en une seule journée vers ces grands centres, et même vers des centres plus éloignés comme Chicago et Winnipeg. 


\subsection{Les régions diversifiées à centre urbain}

Retour à la table des matières

On trouve dans la province de Québec un certain nombre de villes moyennes qui réunissent des entreprises parfois anciennes parfois nouvelles, plus ou moins diversifiées, entreprises qui fournissent aux villes en question et à leur zone d'influence une prospérité non moins enviable que celle de la région de Montréal. Certaines de ces villes sont très dynamiques, leur population jouit d'un niveau de vie très élevé. Mentionnons par exemple Sherbrooke, Trois-Rivières, Chicoutimi, Shawinigan, etc. On peut considérer ces villes comme autant de capitales régionales. Elles ne peuvent que souffrir de la concentration excessive de l'activité économique à Montréal. Elles perdent graduellement leur rôle de centres de décision. À l'examen en effet, on se rend compte qu'il nexiste pas de véritable polarisation autour de ces villes puisqu'une bonne partie des relations que les entreprises, mêmes importantes, qui sont situées dans leur zone d'influence doivent entretenir avec des centres plus importants le sont avec Montréal ou avec Québec, c'est-à-dire passant en réalité pardessus ces capitales régionales. Vous avez remarqué que je n'ai pas mentionné la ville de Québec, qu'on ne peut certes pas assimiler à ces capitales régionales, puisqu'elle possède, du moins en puissance beaucoup des caractéristiques d'un pôle majeur d'attraction. Il n'en reste pas moins vrai que la région de Québec ne manifeste pas tout le dynamisme qu'on pourrait souhaiter.

\subsection{Les régions périphériques}

Un troisième type de régions pourrait être qualifié de périphériques parce qu'elles sont victimes d'un éloignement plus ou moins considérable des principaux centres de développement, cet éloignement étant considéré comme leur principal handicap en matière de croissance économique. Ces régions toutefois sont de deux sortes : on trouve d'une part des régions fort anciennes dont la situation se révèle de plus en plus pénible. Elles doivent compter à la fois sur une 
aide extérieure et sur une émigration massive de leur population pour être en mesure de conserver malgré tout un certain niveau de vie. Et très souvent en dépit de cette émigration un chômage structurel élevé persiste. Ces régions qui ne bénéficient guère do nouveaux investissements, sont dans une situation d'extrême dépendance à l'égard des autres. Dépendantes non seulement du point de vue de l'apport des capitaux nouveaux et des initiatives nouvelles, mais dépendantes même du point de vue de l'apport des denrées les plus courantes de consommation. Ce sont en réalité ces régions qui ont le plus souffert de l'extrême concentration d'activité économique dans les villes les plus importantes. On peut les appeler déprimées, signifiant par là qu'elles ont eu leur heure de prospérité à une certaine époque.

Mais il existe dans les zones périphériques un autre type de régions dont les caractéristiques sont tout à fait différentes : ce sont des régions centrées sur une activité moderne de grande envergure, type Murdochville par exemple ou Thetford- Mines ou BaieComeau ou Arvida. La plupart du temps, ces régions bénéficient d'un niveau de vie relativement élevé dans la mesure où les entreprises en question sont très prospères et disposent de vastes marchés extérieurs. Ce sont malgré tout des régions sous-développées en ce sens qu'elles n'ont pas encore atteint le stade du développement harmonieux et équilibré. Elles sont créées grâce à lactivité d'une industrie motrice, mais ce ne sont pas des régions motrices. On se fait illusion en effet en croyant que la simple installation dans une région désertique et éloignée d'une industrie moderne, même gigantesque est un facteur de polarisation d'activité. On pourrait multiplier les exemples de régions minières et de régions forestières qui réussissent assez bien à tirer leur épingle du jeu mais dans lesquelles aucune activité nouvelle n'est venue se greffer sur l'implantation initiale. Les seules initiatives, qui sont d'ailleurs purement satellites, l'ont été dans le secteur tertiaire sous forme d'installations de restaurants, d'hôtels, de cinémas, de commerces de toutes sortes pour desservir la population locale. Donc peu d'activités de nature industrielle fonctionnant pour le marché régional ou le marché national. Et même si les régions en question sont génératrices de richesse, celles-ci cependant seront largement transférées dans d'autres régions. Ces richesses ne constituent pas 
de point de départ pour un auto-développement. Ce sont des régions en d'autres termes qui ne sont pas autonomes. Elles sont aussi dépendantes que les précédentes et leur économie est extrêmement instable puisqu'elle dépend d'une spécialisation très poussée d'activités économiques.

L'examen des caractéristiques respectives de ces diverses régions nous amène à constater que le déséquilibre qui nous préoccupe est un déséquilibre à la fois structurel et fonctionnel dont les disparités de revenu et de conditions de vie ne sont en réalité que l'une des manifestations. Sur le plan de l'initiative en matière économique, il faut constater l'incapacité d'un très grand nombre de régions de la Province de générer par elles-mêmes des innovations fécondes appuyées sur des ressources régionales. En effet beaucoup de régions qui possèdent de la main-d'œuvre, des matières premières et des capitaux doivent se résoudre à les exporter au bénéfice des pôles d'attraction ayant déjà une taille considérable. Par contre, l'initiative en matière de développement sera d'origine externe.

Voilà sur le plan économique, la source même des déséquilibres, qui trouve son expression la plus frappante dans la comparaison entre la situation de la zone métropolitaine de Montréal et celle du premier type de régions périphériques, celles dont l'économie est déprimée ou retardée. En l'absence d'intervention, les déséquilibres en question sont le résultat d'un double mouvement, l'un faisant bénéficier les régions favorisées de tous les avantages du progrès technique, l'autre au contraire contribuant à appauvrir sans cesse les régions défavorisées.

Nous inspirant d'un économiste français, Raymond Barre, résumons les manifestations de cet effet d'appauvrissement de la façon suivante :

- émigration des éléments jeunes et actifs de la population ;

- émigration des capitaux, le système bancaire captant les épargnes des régions pauvres pour les orienter vers les régions en plein essor, 
- disparition des industries concurrencées par celles des régions développées, qui disposent de marchés plus vastes et travaillent dans la zone des rendements croissants ;

- régression de l'agriculture, qui demeure l'activité prédominante, mais dont le niveau de productivité est en baisse ;

- insuffisance des services publics (routes, voies ferrées, services sociaux).

C'est le fameux cercle vicieux de la misère !

Voilà, certes, un tableau bien noir. D'autant plus noir que, compte tenu des avantages naturels des grandes agglomérations, on ne voit pas bien comment on pourrait favoriser une certaine décentralisation ou du moins freiner le mouvement actuel de concentration, sans introduire dans l'aménagement du territoire des contraintes extérieures, des mécanismes d'aide plus ou moins artificiels.

Je serai le premier à admettre qu'on sacrifie éventuellement un peu de rationalité économique ou une politique de décentralisation qui se réclamerait d'objectifs d'un autre ordre. Mais je ne veux pas entrer sur ce terrain. Je me contenterai ici de soulever un autre point, ou plutôt d'attaquer un argument souvent utilisé par les protagonistes de la concentration, sous prétexte de rationalité économique. On dit souvent en effet que les mécanismes du marché libre se chargent de trouver localisation idéale pour les entreprises nouvelles, celles-ci se dirigeant là où les coûts d'implantation sont les plus faibles.

Cet argument serait acceptable, du moins sur le plan économique, si les entreprises en question et par conséquent la région à laquelle elles appartiennent supportaient la totalité des frais de localisation. Si les dirigeants d'entreprise, dit-on après calcul serré de leurs coûts d'implantation, et compte tenu des coûts de transferts le cas échéant, décident de s'installer dans une agglomération comme Montréal, c'est qu'ils y trouvent leur avantage, et qu'on ne peut pas leur nier le droit d'utiliser la localisation la plus pratique pour eux, et la d'autant plus que l'ensemble de la collectivité ne peut que bénéficier elle-même d'un effort de minimisation des 
coûts. Un tel raisonnement cependant ne tient pas compte du fait que dans les calculs auxquels nous faisons allusion seulement une partie des coûts sociaux de cette implantation, coûts qui peuvent se manifester de différentes façons: par la pollution de l'air par exemple ou la pollution de l'eau ou par un chômage structurel qui serait la conséquence fatale dans d'autres régions d'un excès de concentration dans la région favorisée. En définitive d'ailleurs, les entreprises en question font souvent un mauvais calcul sans s'en rendre compte puisqu'elles sont obligées éventuellement de supporter une partie importante des coûts sociaux en cause, par le truchement de la fiscalité grâce à laquelle une certaine péréquation de revenus sera réalisée au bénéfice des régions pauvres. Ce n'est que grâce à une comptabilité sociale de type global qu'on est en mesure d'évaluer réellement les avantages comparés de la localisation industrielle dans les régions riches et les régions pauvres.

\section{LES ATTITUDES POSSIBLES}

$\underline{\text { Retour à la table des matières }}$

En présence de cette situation caractérisée par des déséquilibres qui tendent à se perpétuer, quelles sont les attitudes possibles ? Nous référons, ici, à la fois, à des attitudes que l'on peut identifier à l'heure actuelle ou à d'autres attitudes qu'il faudrait envisager. Il ne s'agit pas à ce stade-ci de prôner une politique régionale. Il est opportun cependant de prendre conscience dès maintenant des diverses avenues qui s'offrent aux responsables de la planification économique. Car il ne semble pas faire de doute que la plus grande partie de l'opinion n'accepte pas allègrement la situation présente et est donc disposée à ce qu'on applique la thérapie la plus opportune.

Posons d'abord comme principe que l'activité économique doit être répartie sur le territoire de façon à minimiser les coûts de production et à permettre d'obtenir des ressources disponibles le meilleur rendement global possible. Une fois obtenue la masse des 
biens et des services, ceux-ci doivent être éventuellement répartis entre la population de façon équitable et de façon à affecter le moins possible la productivité des différents agents de cette production. On sait que la poursuite simultanée de ces deux objectifs, de production maximale et de répartition optimale oblige parfois à faire des concessions sur le plan de la productivité pure. Mais celle-ci en réalité ne constitue pas un objectif par elle-même.

Quand on essaie d'appliquer ces principes à un territoire déjà occupé depuis des générations, avec une contexture donnée, bonne ou mauvaise, diverses contraintes s'imposent dans une politique de mise en valeur du territoire. Premièrement, contraintes qui se manifesteront par l'obligation tenir compte de la répartition initiale de la population même si le peuplement a été fait de façon tout à fait irrationnelle. Deuxièmement, de la dispersion des différentes ressources naturelles dans la mesure où celles-ci peuvent constituer des facteurs de localisation d'activité économique ; troisièmement, de l'existence d'investissement sociaux sous forme d'écoles, d'églises ou de bâtiments publics de toutes sortes, investissements qui normalement doivent être amortis sur une période assez longue.

Dans l'élaboration d'une politique régionale, plusieurs formules sont possibles. Pour les uns il faudrait encourager ou du moins ne pas contrecarrer la tendance à la concentration de l'activité économique dans la région métropolitaine de Montréal puisque cette région réunit à l'heure actuelle, dit-on, la plupart des facteurs favorables à la localisation de l'activité économique. Ce serait, comme nous l'avons vu plus précédemment, le milieu social qui conviendrait le mieux aux dirigeants économiques, un milieu jouissant d'une très haute productivité. Un tel raisonnement cependant, nous l'avons vu précédemment, pèche d'une certaine façon puisqu'il ne tient pas compte de l'ensemble des facteurs, par exemple la nécessité onéreuse de réaliser certains travaux publics de grande envergure qui ne deviennent indispensables que si l'agglomération dépasse un certain seuil de développement. Corollaire inéluctable d'une telle attitude : c'est qu'on accepte implicitement une politique de transferts économiques et sociaux de nature à insuffler une vitalité plus ou moins factice aux régions déshéritées. Ce qui, ne 
l'oublions pas, absorbe une partie des avantages économiques tirés présumément de la concentration.

Si l'on refuse cette solution qui consiste à accepter l'évolution telle qu'elle se pratique à l'heure actuelle d'autres formules sont possibles.

Le déplacement graduel et ordonné de la population des régions périphériques vers les régions centrales. Une telle politique cependant se heurte à des difficultés d'ordre social majeures qui font quil est très difficile d'y songer, du moins en tant que politique globale. Car, sur un plan plus partiel, et dans certaines zones restreintes, une telle formule s'impose de toute nécessité, surtout comme complément à des politiques de développement harmonisé.

Une deuxième solution consiste dans ce qu'on a appelé le conservatisme démographique, politique qui se formulerait ainsi : dans la mesure où l'on trouve dispersés, sur un territoire aussi vaste que celui de la province de Québec, différents noyaux plus ou moins importants de population, il faut absolument, par une politique appropriée, créer des activités permettant l'utilisation, sur place, de ces diverses ressources en main-d'œuvre. L'initiative, ici, consiste donc à déplacer les activités économiques et à les orienter vers les endroits où la population est déjà installée. Une telle politique prête flanc à la critique parce qu'elle tend à perpétuer les erreurs du passé. Elle repose au fond sur l'idée désuète qu'à peu près n'importe quelle région, quels que soient ses atouts, peut servir de zone d'accueil pour l'industrie secondaire ou le commerce.

Il existe enfin une troisième possibilité qui est la création ou plutôt le développement de certains pôles intermédiaires c'est-à-dire de villes qui possèdent à un degré satisfaisant une vocation pour la localisation industrielle. Il s'agit d'intensifier alors l'implantation de nouvelles activités, au besoin par des mesures d'assistance au début. Mais après un certain temps, ces villes dépassent un certain seuil de développement qui les transforment en zones de polarisation, capables de générer par elles-mêmes de nouvelles activités économiques, et, au surplus, de propager leur dynamisme dans la région 
avoisinante, selon des politiques intermédiaires, entre la concentration expressive telle qu'elle existe à l'heure actuelle, et la décentralisation aussi excessive qui consisterait en une politique de saupoudrage de petits établissements dispersés dans toute la Province. Selon cette dernière formule, il serait désirable de choisir un certain nombre de centres privilégiés possédant déjà des atouts considérables, ce qui suppose nécessairement l'abandon de tout esprit de clocher, qui a tellement nui dans le passé à un aménagement rationnel du territoire.

Je n'insiste pas sur les modalités d'une telle politique, que pour ma part je choisis sans ambages. Elle m'apparaît comme un compromis tout à fait honorable entre les impératifs de la plus haute productivité dont on ne peut tout de même pas se désintéresser, puisque la province de Québec ne constitue pas un marché isolé en Amérique du Nord, et une politique de régénérescence des régions qui peut fort bien se justifier par de multiples facteurs autres qu'économiques.

\section{Roland Parenteau}

Président du Conseil d’orientation économique du Québec

Fin du texte 